\title{
Tuning the Magnetic Anisotropy at a Molecule-Metal Interface
}

Bairagi, K.; Bellec, A.; Repain, V.; Chacon, C.; Girard, Y.; Garreau, Y.; Lagoute, J.; Rousset, S.; Breitwieser, R.; Hu, Yu-Cheng

Total number of authors:

15

Published in:

Physical Review Letters

Link to article, DOI:

10.1103/PhysRevLett.114.247203

Publication date:

2015

Document Version

Publisher's PDF, also known as Version of record

Link back to DTU Orbit

Citation (APA):

Bairagi, K., Bellec, A., Repain, V., Chacon, C., Girard, Y., Garreau, Y., Lagoute, J., Rousset, S., Breitwieser, R., Hu, Y-C., Chao, Y. C., Pai, W. W., Li, D., Smogunov, A., \& Barreteau, C. (2015). Tuning the Magnetic Anisotropy at a Molecule-Metal Interface. Physical Review Letters, 114(24), [247203].

https://doi.org/10.1103/PhysRevLett.114.247203

\section{General rights}

Copyright and moral rights for the publications made accessible in the public portal are retained by the authors and/or other copyright owners and it is a condition of accessing publications that users recognise and abide by the legal requirements associated with these rights.

- Users may download and print one copy of any publication from the public portal for the purpose of private study or research.

- You may not further distribute the material or use it for any profit-making activity or commercial gain

- You may freely distribute the URL identifying the publication in the public portal 


\title{
Tuning the Magnetic Anisotropy at a Molecule-Metal Interface
}

\author{
K. Bairagi, ${ }^{1}$ A. Bellec, ${ }^{1}$ V. Repain, ${ }^{1,}$ C. Chacon, ${ }^{1}$ Y. Girard, ${ }^{1}$ Y. Garreau, ${ }^{1}$ J. Lagoute, ${ }^{1}$ S. Rousset, ${ }^{1}$ R. Breitwieser, ${ }^{2}$ \\ Yu-Cheng Hu, ${ }^{2}$ Yen Cheng Chao, ${ }^{2}$ Woei Wu Pai ${ }^{2,4}$ D. Li, ${ }^{3}$ A. Smogunov, ${ }^{3}$ and C. Barreteau ${ }^{3,5}$ \\ ${ }^{1}$ Laboratoire Matériaux et Phénomènes Quantiques, Université Paris Diderot-Paris 7, UMR CNRS 7162, \\ 10 rue Alice Domon et Léonie Duquet 75205 Paris Cedex 13, France \\ ${ }^{2}$ Center for Condensed Matter Sciences, National Taiwan University, Taipei 106 Taiwan, Republic of China \\ ${ }^{3}$ Service de Physique de l'Etat Condensé (CNRS UMR 3680), IRAMIS/SPEC, CEA Saclay, F-91191 Gif-sur-Yvette Cedex, France \\ ${ }^{4}$ Department of physics, National Taiwan University, Taipei 106, Taiwan, Republic of China \\ ${ }^{5}$ DTU NANOTECH, Technical University of Denmark, Ørsteds Plads 344, DK-2800 Kgs. Lyngby, Denmark
}

(Received 26 January 2015; published 16 June 2015)

\begin{abstract}
We demonstrate that a $\mathrm{C}_{60}$ overlayer enhances the perpendicular magnetic anisotropy of a Co thin film, inducing an inverse spin reorientation transition from in plane to out of plane. The driving force is the $\mathrm{C}_{60} / \mathrm{Co}$ interfacial magnetic anisotropy that we have measured quantitatively in situ as a function of the $\mathrm{C}_{60}$ coverage. Comparison with state-of-the-art $a b$ initio calculations show that this interfacial anisotropy mainly arises from the local hybridization between $\mathrm{C}_{60} p_{z}$ and $\mathrm{Co} d_{z^{2}}$ orbitals. By generalizing these arguments, we also demonstrate that the hybridization of $\mathrm{C}_{60}$ with a $\mathrm{Fe}(110)$ surface decreases the perpendicular magnetic anisotropy. These results open the way to tailor the interfacial magnetic anisotropy in organic-material-ferromagnet systems.
\end{abstract}

DOI: 10.1103/PhysRevLett.114.247203

PACS numbers: 75.70.Cn, 73.20.Hb, 75.30.Gw, 78.20.Ls

The use of organic materials in spintronic devices has recently raised a lot of interest. Large spin diffusion time in organic materials combined with complex couplings at the interfaces lead to very large magnetoresistance $[1,2]$. $\mathrm{C}_{60}$ is one of the model molecules that has been used so far to evidence the room temperature magnetoresistive effect for relatively thick layers [3]. More recently, peculiar interactions between a molecular layer and a cobalt electrode allowed for the demonstration of magnetoresistive behavior with a single magnetic electrode [4]. Despite the numerous transport measurements reported in various molecular spin-valve devices, only little is known on the effect of organic-material-ferromagnetic interfaces on the device performance, especially regarding magnetic anisotropy energy (MAE) [5].

It is known that MAE of a thin magnetic layer can be seriously affected by the interface with nonmagnetic layers. The influence of carbon based materials was believed to be small because of their low spin-orbit coupling constant but recent works have pointed out that the out-of-plane MAE is enhanced at a graphene-Co interface [6-8]. However, a quantitative measurement and the understanding of such an influence of carbon based overlayers on the MAE is still missing.

In this Letter, we demonstrate for the first time by in situ and real time control of thin film magnetic properties under a molecular deposition that a strong interfacial magnetic anisotropy can increase $\left[\mathrm{C}_{60} / \mathrm{Co}(0001)\right]$ or decrease $\left[\mathrm{C}_{60} / \mathrm{Fe}(110)\right]$ the perpendicular magnetic anisotropy (PMA). These results are analyzed and explained by state-of-the-art $a b$ initio calculations where we decompose the magnetic anisotropy on the different $d$ orbitals of $\mathrm{Co}$ and Fe. The favored hybridization between metallic $d_{z^{2}}$ and carbon $p_{z}$ orbitals at the interface explains the experimental findings and gives a simple and predictive view of the interfacial magnetic anisotropy between a $3 d$ ferromagnet and an organic layer, which is of crucial importance for the future development of organic spintronics.

An in situ ultrahigh vacuum polar magnetooptical Kerr effect (MOKE) setup was used to measure magnetic cycles during the deposition of $\mathrm{C}_{60}$ on $\mathrm{Co} / \mathrm{Au}(111)$ and $\mathrm{Fe} / \mathrm{Au}(111)$ ultrathin films. The magnetic cycles were recorded every $20 \mathrm{~s}$ with a magnetic field applied parallel to the surface normal with a sweep rate of $1 \mathrm{~Hz}$ generating a maximum field of $68 \mathrm{mT}$. It is known that $\mathrm{Co}$ on $\mathrm{Au}(111)$ undergoes a spin reorientation transition (SRT) from out of plane to in plane at a Co thickness $t_{c}$ of $4.2 \mathrm{ML}[9,10]$.This value was used as a calibration of our Co thickness, in good agreement with STM and Auger electron spectroscopy results. To calibrate the thickness of $\mathrm{C}_{60}$ molecules, we used scanning tunneling microscopy (STM) images of submonolayer deposition on the $\mathrm{Au}(111)$ surface [11]. A monolayer (ML) of $\mathrm{C}_{60}$ is therefore defined as a dense hexagonal packing obtained on $\mathrm{Au}(111)$ [12]. However, STM images of $\mathrm{C}_{60}$ on the Co film (cf. top inset of Fig. 1) show a defective layer with a less dense packing. Therefore, analyzing the molecular surface density, we found that the Co surface is fully covered by $\mathrm{C}_{60}$ around $0.75 \pm 0.05 \mathrm{ML}$.

To study the change in magnetism of Co films upon molecule deposition, experiments were performed both below and above $t_{c}$. The experiment summarized in Fig. 1 


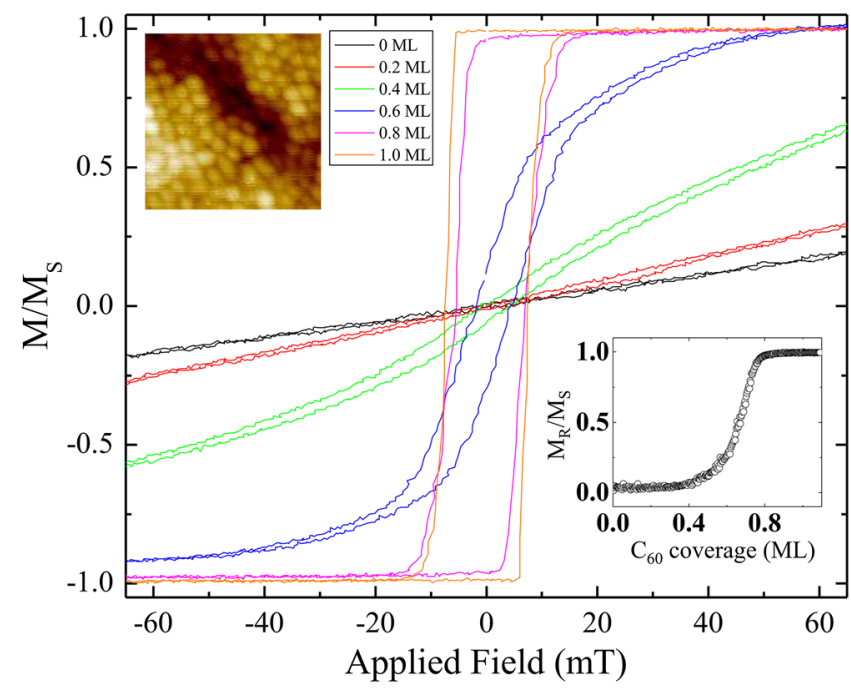

FIG. 1 (color online). Raw hysteresis cycles measured during the deposition of $\mathrm{C}_{60}$ on $5.5 \mathrm{ML} \mathrm{Co} / \mathrm{Au}(111)$ in a polar MOKE configuration. Bottom inset: Graph of the remanent magnetization $M_{R}$ over the saturated magnetization $M_{S}$ as a function of the $\mathrm{C}_{60}$ thickness. Top inset: Typical STM image after the deposition of $1 \mathrm{ML}$ of $\mathrm{C}_{60}$ on $5.5 \mathrm{ML} \mathrm{Co} / \mathrm{Au}(111)$ $\left(12 \times 12 \mathrm{~nm}^{2}, 1.0 \mathrm{~V}, 20 \mathrm{pA}\right)$.

on a 5.5 ML Co film demonstrates that the magnetization switches from in plane to out of plane, showing a significant change in MAE with $\mathrm{C}_{60}$ coverage (cf. movie, Ref. [11]). The bottom inset shows the squareness $\left(M_{R} / M_{S}\right.$, i.e., ratio of the remanent magnetization over saturated magnetization) of the cycles as a function of the $\mathrm{C}_{60}$ coverage, inducing an abrupt inverse SRT. Thus the $\mathrm{C}_{60}$ layer induced a PMA that needs to be quantified and understood.

Another indirect observation of this rise in MAE is the magnetic behavior of a 3.2 ML Co film that keeps an out-of-plane magnetization with the $\mathrm{C}_{60}$ overlayer but shows a modified coercive field $H_{c}$. Results are summarized in Fig. 2(a), which displays an increase of $H_{c}$ by a factor larger than two with $\mathrm{C}_{60}$ coverage. This increase in coercivity is indirectly related to an increase of the out-ofplane magnetic anisotropy, although a quantitative determination is difficult because magnetic exchange could also play a role [13]. We also plot in Fig. 2(a) the variation of the saturated MOKE signal as a function of the $\mathrm{C}_{60}$ coverage. Assuming a constant magnetooptical constant, this can be interpreted as a slight decrease of the total Co film magnetic moment (given in absolute value measured independently by x-ray magnetic circular dichroism, [11]) [14].

To obtain a quantitative determination of the MAE variation with $\mathrm{C}_{60}$ deposition, we have performed hard axis magnetometry for Co films well above $t_{c}$, i.e., still in plane with a full fullerene layer. In this case, the saturation field is given by the anisotropy field $H_{K}=2 K_{\text {eff }} / \mu_{0} M_{S}$ where $K_{\text {eff }}$ is the total effective anisotropy of the Co film.
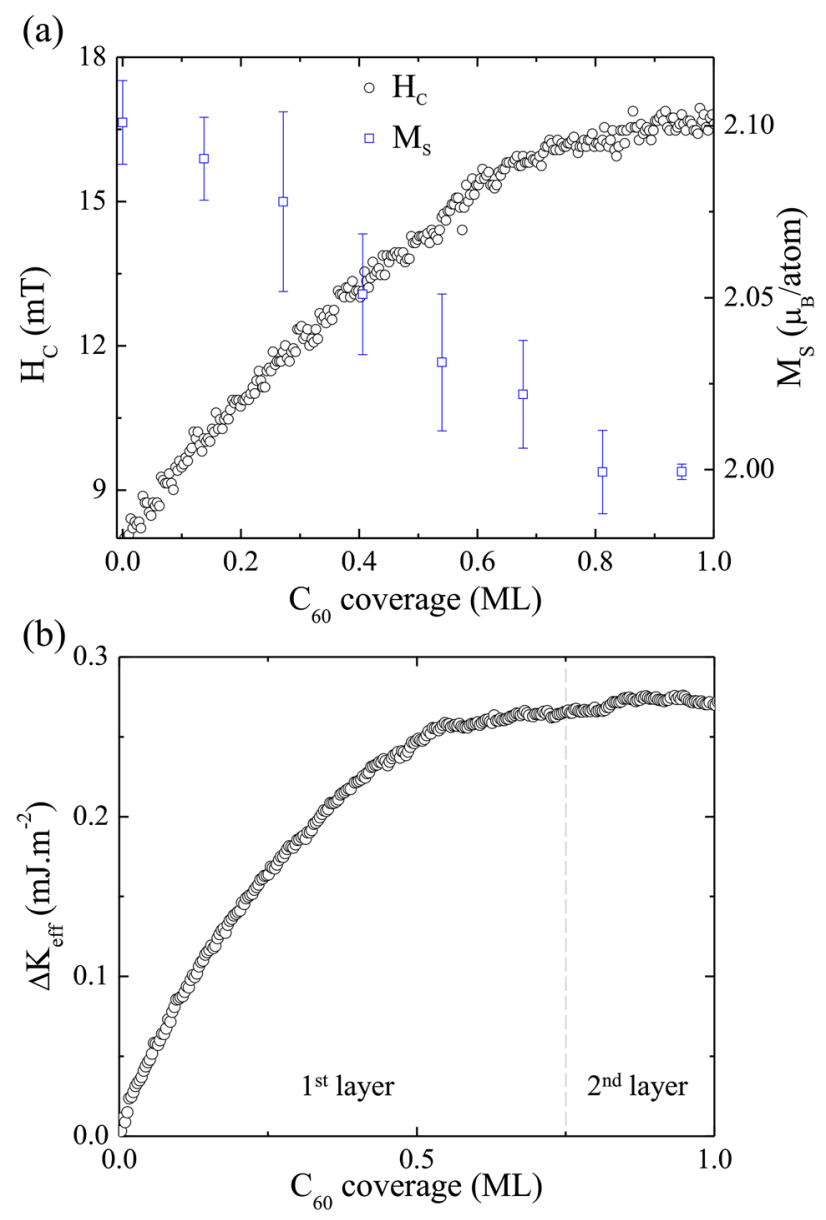

FIG. 2 (color online). (a) Variation of $H_{C}$ and $M_{S}$ as a function of $\mathrm{C}_{60}$ coverage for a $3.2 \mathrm{ML}$ Co film. (b) Interfacial magnetic anisotropy variation with $\mathrm{C}_{60}$ coverage for a $6.4 \mathrm{ML}$ Co film.

As our in situ magnetic field is not high enough to saturate these reversible cycles, we extract $H_{K}$ from the slope of the individual in-plane cycles and the saturation magnetization value. The latter is calibrated by an extrapolation of the saturated cycles measured for Co thickness below $t_{c}$. We find for our $\mathrm{Co} / \mathrm{Au}(111)$ system, a surface anisotropy term $K_{S}=0.6 \pm 0.1 \mathrm{~mJ} \mathrm{~m}^{-2}$ and a volume anisotropy $K_{V}=$ $-800 \pm 100 \mathrm{~kJ} \mathrm{~m}^{-3}$ [11], in good agreement with the literature $[10,15]$. The change of MAE, expressed in surface anisotropy units, is plotted as a function of the $\mathrm{C}_{60}$ coverage for a $6.4 \mathrm{ML}$ Co film in Fig. 2(b). This variation is linear with the coverage at low coverage and saturates around $0.7 \mathrm{ML}$. This corresponds exactly to the completion of a full fullerene layer, as determined by STM. For higher coverage, $\mathrm{C}_{60}$ molecules adsorb on a second layer, without direct interaction with Co atoms. We can therefore only ascribe this MAE change to an interface effect induced by the hybridization between $\mathrm{C}_{60}$ and $\mathrm{Co}$. The interface anisotropy is around $0.3 \mathrm{~mJ} \mathrm{~m}^{-2}$, i.e., around a half of the $\mathrm{Co} / \mathrm{Au}$ anisotropy. This value is not negligible, especially if one considers that only a small fraction of the 
(a)
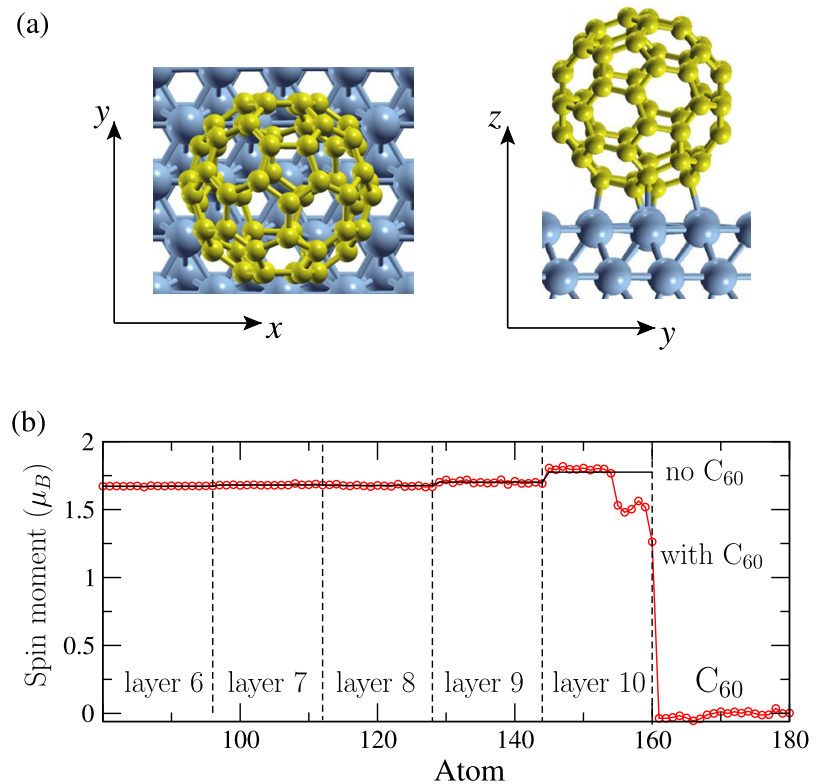

(c)

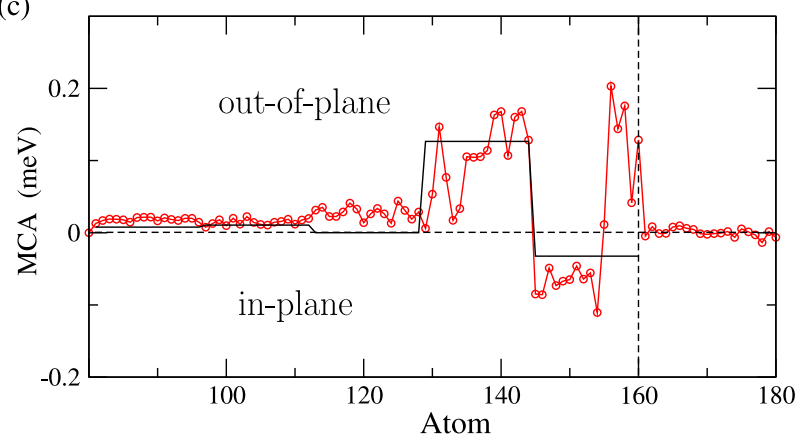

FIG. 3 (color online). DFT results for a 10-layer Co slab covered with $\mathrm{C}_{60}$ molecules: (a) lowest energy adsorption configuration of $\mathrm{C}_{60}$ monolayer; (b) atomic spin moments for the Co slab with (red open circles) and without (black solid curve) $\mathrm{C}_{60}$ molecules. There are $16 \mathrm{Co}$ atoms per layer due to the $4 \times 4$ in-plane periodicity. Only contributions from the five outer $\mathrm{Co}$ planes close to $\mathrm{C}_{60}$ monolayer are shown; (c) atomically resolved MCA for the same two cases as in (b).

surface atoms are hybridized due to the spherical geometry of the $\mathrm{C}_{60}$ molecule [ 6 over 16 for a dense packing as shown in Fig. 3(a)] and that carbon is a light material with a low spin-orbit coupling constant. In the following, we give an atomic scale interpretation of this interfacial anisotropy in light of $a b$ initio calculations.

We have performed density functional theory (DFT) calculations using the plane-wave electronic structure package QUANTUM-ESPRESSO [16]. Since the realistic atomic structure of $\mathrm{Co}$ films on a $\mathrm{Au}(111)$ substrate is experimentally complex and not perfectly known, we have chosen to simulate this system by a thick enough hcp Co slab containing 10 atomic layers with $\mathrm{C}_{60}$ molecules adsorbed on one side of the slab (possible effects of the underlying Au substrate are thus neglected for simplicity). A $4 \times 4$ in-plane periodicity is used resulting in $\mathrm{C}_{60}-\mathrm{C}_{60}$ in-plane separation between the closest carbon atoms of about $3.2 \AA$. Our goal is to look at the local change in magnetic anisotropy of $\mathrm{Co}$ atoms close to the $\mathrm{C}_{60}$ molecular layer. Such a thick 10-layer Co slab is necessary to reduce finite-size effects. In particular, the magnetic anisotropy of surface Co atoms was found to be rather sensitive to the slab thickness, both in sign and magnitude, as demonstrated for 5-, 10-, and 15-layer Co slabs [11].

To determine the adsorption geometry of $\mathrm{C}_{60}$ molecules we performed atomic relaxations using a thinner 5-layer $\mathrm{Co}$ slab. In both cases with and without $\mathrm{C}_{60}$ on the Co slab, the first three layers were fixed at their bulk positions while the outer layers were allowed to relax to minimize the total energy.

The most stable configuration shown in Fig. 3(a) corresponds to $\mathrm{C}_{60}$ molecules bound by a pentagonhexagon edge to a Co surface atom [14]. Figure 3(b) shows atomic spin moments for a clean 10-layer Co slab (black curve) and for the same slab covered with $\mathrm{C}_{60}$ molecules (red open circles). Since the influence of the $\mathrm{C}_{60} /$ Co interface is vanishingly small after four Co layers, we only display the results for the five outer Co layers. One can clearly see that the Co magnetism is much affected by $\mathrm{C}_{60}$, similarly to $\mathrm{Cr}$ [17] and $\mathrm{Fe}$ [18]. In particular, spin moments of the six Co atoms making bonds with $\mathrm{C}_{60}$ (Co atoms numbered as 155-160) are strongly reduced, dropping down to $\sim 1.25 \mu_{B}$ for the atom just beneath the $\mathrm{C}_{60}$ (Co atom number 160). On the contrary, we find that the $\mathrm{C}_{60}$ molecule gets slightly polarized with $-0.23 \mu_{B}$ in total [17].

We define MAE as the difference in total energies between in-plane and out-of-plane magnetic configurations, $E_{\|}-E_{\perp}$. The total MAE has two contributions, namely: (i) magnetocrystalline anisotropy (MCA) due to spin-orbit coupling (SOC) and (ii) shape anisotropy due to dipole-dipole magnetic interactions. We first discuss the MCA and will briefly address the shape anisotropy at the end of this section. In our DFT calculations, the spin-orbit coupling, crucial for MCA, is taken into account via fully relativistic pseudopotentials which are generated by solving the atomic Dirac equation for each atomic type. The MCA is then calculated within the force theorem approach, as implemented recently in Ref. [19].

In Fig. 3(c) we present atomically resolved MCA. For a clean slab (black line), the MCA values from the five outer planes sum up to the total value of $\approx 1.8 \mathrm{meV}$, thus favoring the out-of-plane magnetization direction. Upon adsorption of $\mathrm{C}_{60}$ molecules (red open circles), the overall out-of-plane MCA is further enhanced by $\approx 0.9 \mathrm{meV}$. Importantly, this enhancement is mainly provided by the six Co atoms directly involved in $\mathrm{Co}_{0} \mathrm{C}_{60}$ bonding (numbered as 155-160) that show locally an increase of anisotropy by about $0.2 \mathrm{meV} /$ atom, which is a quite significant value. Their bonding to $\mathrm{C}_{60}$ has thus a very strong effect reversing their preferable magnetization axis from the in-plane to out-of-plane direction. 
(a)

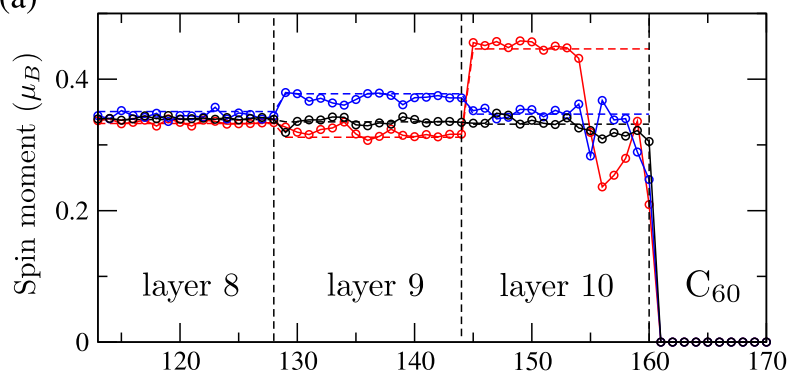

(b)

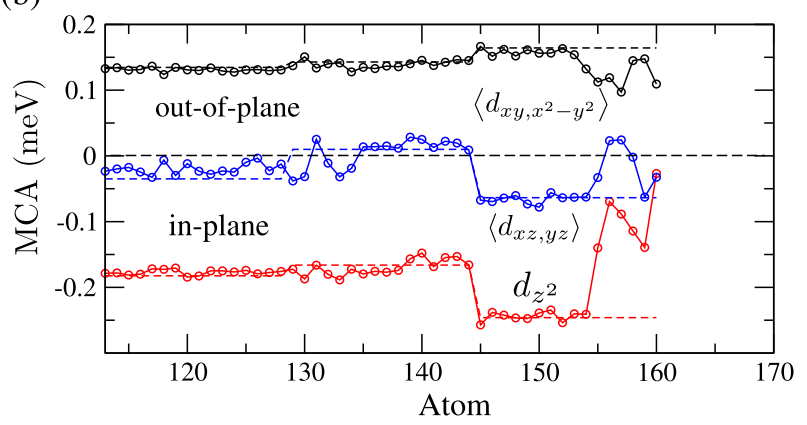

FIG. 4 (color online). $d$ orbital decomposition of (a) atomic spin moments and (b) MCA for the 10 layers Co slab, pure (dashed lines) and covered with $\mathrm{C}_{60}$ molecules (solid lines). The results for three Co layers close to $\mathrm{C}_{60}$ molecules are only presented. Because of symmetry, contributions from different orbitals in $\left\{d_{x z, y z}\right\}$ and $\left\{d_{x y, x^{2}-y^{2}}\right\}$ pairs are very similar so that their averaged values are presented for simplicity.

In order to interpret the influence of $\mathrm{C}_{60}$ on local magnetism, we present spin moments in Fig. 4(a) and MCA in Fig. 4(b) decomposed over the different Co $d$ orbitals (only the data for three Co layers close to $\mathrm{C}_{60}$ are shown for simplicity) for the bare $\mathrm{Co}$ and $\mathrm{C}_{60}$ on Co. Interestingly, from Fig. 4(b) we notice that for a pure Co slab, differently oriented $d$ orbitals favor different magnetic orientations. In particular, the $d_{x y, x^{2}-y^{2}}$ orbitals, parallel to the slab surface, show strong out-of-plane anisotropy, while the $d_{z^{2}}$ orbital, perpendicular to the slab, favors the in-plane orientation (the other $d_{x z, y z}$ orbitals show intermediate values). Upon $\mathrm{C}_{60}$ adsorption, all the contributions at $\mathrm{Co}$ atoms bound to $\mathrm{C}_{60}$ get suppressed in magnitude. However, the degree of this reduction depends on the hybridization strength of the corresponding orbital with the $\mathrm{C}_{60}$ states: evidently, it is the strongest for the outof-plane oriented $d_{z^{2}}$ orbital and the smallest for the inplane $d_{x y, x^{2}-y^{2}}$ states. As a result of this unbalance, the overall MCA for these Co atoms appears to enhance strongly, favoring the out-of-plane orientation. The same argument is valid for spin moments: their reduction at Co atoms bound to $\mathrm{C}_{60}$ is essentially due to the suppression of the $d_{z^{2}}$ contribution, as clearly demonstrated in Fig. 4(a).

Finally, we discuss shape anisotropy, the other contribution to magnetic anisotropy, which favors the in-plane magnetic orientation. It is calculated directly from atomic spin moments as those presented in Fig. 3(b) [11]. For a 5-layer Co film, we find a value of $-7.5 \mathrm{meV}$ per $4 \times 4$ unit cell, that drops to $-6.9 \mathrm{meV}$ when covered with $\mathrm{C}_{60}$. This decrease of the shape anisotropy magnitude is perfectly consistent with the decrease of the mean magnetization calculated in Fig. 3(b).

Quantitatively, we find for a 5 ML slab containing $16 \mathrm{Co}$ atoms per atomic plane an overall increase of the out-of-plane magnetic anisotropy of $1.5 \mathrm{meV}(0.9 \mathrm{meV}$ for magnetocrystalline and $0.6 \mathrm{meV}$ for dipolar anisotropies), i.e., around $19 \mu \mathrm{eV} /$ atom if homogeneously averaged over all the Co atoms. However, this magnetic anisotropy increase is mainly due to interfacial atoms, as shown in Fig. 3(c). Averaged over the interfacial layer, it leads to almost $0.1 \mathrm{meV} /$ atom or, converted in international units $\Delta K_{\text {eff }}=0.27 \mathrm{~mJ} \mathrm{~m}^{-2}$, in good agreement with our experimental measurement.

We believe that the theoretical arguments put forward to explain the experimental finding are rather general and can be applied to other metal-molecule interfaces. If the $d_{z^{2}}$ surface component of the MCA favors in-plane (out-of-plane) magnetization, the hybridization with $\mathrm{C}_{60}$ will enhance out-of-plane (in-plane) magnetization. We have therefore performed a series of DFT calculations to determine the atomically and orbital resolved MCA of various bare surface orientations and magnetic elements. We predict that the densest surface of iron [Fe(110)] has an opposite behavior to the $\operatorname{Co}(0001)$ surface since the contribution of the $d_{z^{2}}$ orbital to the surface MCA is clearly out of plane and therefore deposition of $\mathrm{C}_{60}$ should reinforce in-plane anisotropy, which is indeed observed experimentally [11]. Finally let us note that in the case of another type of molecule more orbitals could be involved in the hybridization process which could make the general picture more complicated.

In conclusion, we have shown that a molecular $\mathrm{C}_{60}$ overlayer deposited on a Co thin film surface induces an out-of-plane interfacial anisotropy that is able to give birth to an inverse spin reorientation transition from in-plane to out-of-plane magnetization in the system $\mathrm{Co} / \mathrm{Au}(111)$. The quantitative determination of the magnetization and anisotropy change with the $\mathrm{C}_{60}$ coverage compares well with $a b$ initio calculations. The hybridization between $\mathrm{C}$ atoms and $\operatorname{Co} d_{z^{2}}$ (and, to a lesser extent, $d_{x z, y z}$ ) orbitals is at the origin of the reduction of the spin moment and of the local increase of the out-of-plane anisotropy. Computing the magnetic anisotropy of the surface $d_{z^{2}}$ orbital for different systems, we can predict that the $\mathrm{Fe}(110)$ should show a decrease of PMA upon $\mathrm{C}_{60}$ deposition, as indeed observed experimentally. We believe that these findings are rather general and can apply to all other organic systems showing similar hybridizations.

This work has been funded partly by ANR-BLANC-12 BS10 006 and by the HEFOR project of the Labex SEAM. 
We thank the staff of DRAGON and XPEEM beam lines at the National Synchrotron Radiation Research Center of Taiwan for support. V. R. thanks Institut Universitaire de France for support. D. Li has received funding from the European Research Council under the European Union's Seventh Framework Programme (FP7/2007- 2013)/ERC grant agreement No. 259297. The ab initio calculations were performed using HPC resources from GENCI[TGCC/IDRIS] (Grants No. 2014096813 and No. 2015097416).

*vincent.repain@univ-paris-diderot.fr

[1] C. Barraud et al., Nat. Phys. 6, 615 (2010).

[2] R. Lin, F. Wang, M. Wohlgenannt, C. He, X. Zhai, and Y. Suzuki, Synth. Met. 161, 553 (2011).

[3] M. Gobbi, F. Golmar, R. Llopis, F. Casanova, and L. E. Hueso, Adv. Mater. 23, 1609 (2011).

[4] K. V. Raman et al., Nature (London) 493, 509 (2013).

[5] Y.-J. Hsu et al., J. Phys. Chem. Lett. 4, 310 (2013).

[6] C. Vo-Van et al., New J. Phys. 12, 103040 (2010).

[7] N. Rougemaille, A. T. N'diaye, J. Coraux, C. Vo-Van, O. Fruchart, and A. K. Schmid, Appl. Phys. Lett. 101, 142403 (2012).
[8] R. Decker, J. Brede, N. Atodiresei, V. Caciuc, S. Blügel, and R. Wiesendanger, Phys. Rev. B 87, 041403 (2013).

[9] R. Allenspach, M. Stampanoni, and A. Bischof, Phys. Rev. Lett. 65, 3344 (1990).

[10] G. Rodary, V. Repain, R. L. Stamps, Y. Girard, S. Rohart, A. Tejeda, and S. Rousset, Phys. Rev. B 75, 184415 (2007).

[11] See Supplemental Material at http://link.aps.org/ supplemental/10.1103/PhysRevLett.114.247203 for movie of the raw hysteresis cycles, x-ray magnetic circular dichroism measurements, theoretical details and results on $\mathrm{Fe}(110)$.

[12] E. I. Altman and R. J. Colton, Phys. Rev. B 48, 18244 (1993).

[13] M. Callsen, V. Caciuc, N. Kiselev, N. Atodiresei, and S. Blügel, Phys. Rev. Lett. 111, 106805 (2013).

[14] T. Moorsom et al., Phys. Rev. B 90, 125311 (2014).

[15] C. Chappert and P. Bruno, J. Appl. Phys. 64, 5736 (1988).

[16] P. Giannozzi et al., J. Phys. Condens. Matter 21, 395502 (2009).

[17] S. L. Kawahara, J. Lagoute, V. Repain, C. Chacon, Y. Girard, S. Rousset, A. Smogunov, and C. Barreteau, Nano Lett. 12, 4558 (2012).

[18] T. L. A. Tran, D. Çakir, P. K. J. Wong, A. B. Preobrajenski, G. Brocks, W. G. van der Wiel, and M. P. de Jong, ACS Appl. Mater. Interfaces 5, 837 (2013).

[19] D. Li, C. Barreteau, M. R. Castell, F. Silly, and A. Smogunov, Phys. Rev. B 90, 205409 (2014). 